\title{
Effect of All Fours Belly Breathing Exercise on Aerobic Capacity Among Athletes
}

\author{
Avanianban Chakkarapani*, Wen Yi Yap, How Kuan Ling \\ Department of Physiotherapy, Faculty of Medicine and Health Sciences, Universiti Tunku Abdul Rahman, Malaysia
}

Submission: July 7, 2019; Published: August 06, 2019

*Corresponding author: Avanianban Chakkarapani and Abdul Rahman, Department of Physiotherapy, Faculty of Medicine and Health Sciences, Universiti Tunku, Malaysia

\begin{abstract}
Study Design: Controlled laboratory study, experimental (1 group X 2-time frame) study.

Background: The role of breathing exercise and their effect on a wide range of aspects relevant to orthopedics and sports rehabilitation has become the focus of a growing body of research, yet their effect in sport physical therapy is not well established.

Objectives: To assess the effect of all fours belly breathing exercise on shuttle run performance among athletes.

Methods: A total of 20 participants participated in this study. Group 1(n $=20)$ athletic group. Informed consent and study procedure was explained to all the participants and a pre- shuttle run test (SRT) was assessed. After assessment the participants were engaged in all fours belly breathing exercise for 4 weeks and post SRT was assessed.

Results: The mean SRT score before 4 weeks of all fours belly breathing exercise was 3.305. (Standard deviation $=1.46$ ) The minimum score was 1.143 while the maximum score was 8.182. Whereas the mean SRT score after 4 weeks of all fours belly breathing exercise was 3.95 (Standard deviation $=1.36$ ) The minimum score was 2.5 while the maximum score was 8.364 . Whereas using student $t$ test the mean difference between SRT score before 3.31 (1.47) and SRT score after 3.95 (1.37) is statistically significant ( $\mathrm{p}<0.001)$. As well as the mean difference between predicted V02max before 23.78 (4.90) and predicted V02max after 25.87 (4.74) is statistically significant $(\mathrm{p}<0.001)$

Conclusion: Thus, this study concludes that all fours belly breathing is effective in improving the shuttle run performance among the normal subjects

Keywords: Belly breathing; Shuttle run; Breathing exercise; Quadruped position

Abbreviations: SRT: Shuttle Run Rest; DOMS: Delayed Onset of Muscle Soreness; EMG: Electromyography; ZOA: Zone of Apposition; RA: Rectus Abdominus; IO: Internal Oblique; EO: External Obloque; TA: Transvers Abdominus; CRF: Cardiorespiratory Fitness; METs: Metabolic Equivalents
\end{abstract}

\section{Introduction}

During exercise the ventilation demand keeps on raising the peak, this process defines an increased neural drive to the muscles of respiration. In turn there will be increased mechanical power developed by the muscles. Muscle power always depends on the velocity of shortening and the pressure generated the muscle under contraction. During exercise the diaphragm is primarily a "flow generator". Hence the diaphragm muscle generates mechanical power is mainly expressed as velocity of shortening rather than pressure. Conversely, rib cage and abdominal muscles are primarily "pressure generators", i.e. develop the pressures required to move the rib cage and abdomen, respectively [1]. During exercise the expiratory muscles play an active role in breathing. Within each single breath their action is highly coordinated with the inspiratory muscles. During inspiration, while the rib cage muscles contract, the abdominal muscles gradually relax, and vice versa during expiration. This mechanism has several effects:

i. It prevents rib cage distortion;

ii. The diaphragm is unloaded and can act as a flow generator

iii. The volume of the abdomen is decreased below resting levels $[1,2]$.

As a result, end-expiratory lung volume is decreased during exercise and the mechanics of breathing is optimized for several reasons. Tidal volume occurs in the most compliant part of the respiratory system; the diaphragm is lengthened and thus works near its optimal length; at each breath part of 
the required inspiratory work is previously stored in the form of elastic energy during the previous expiration [3]. Based on the role and physiology of abdominal and diaphragm muscles it is well known that exercise therapist should give significant priority to these muscles. Similarly, the diaphragm, a component of core stability, plays a role in respiration as discussed earlier and trunk stability by controlling intra-abdominal pressure and reducing the stress on the spine through coordinated action with the abdominal and pelvic floor muscles $[4,5]$.

There are still many "experts" who give advice regarding core stabilization but completely fail to mention the diaphragm. There has however, been more frequent mentioning of diaphragm breathing lately. Unfortunately, it often only gets added to trunk stability exercise prescriptions as final comment of "make sure to maintain diaphragm breathing while exercising". It is clearly understood that neuro-muscular activation of diaphragm during trunk stability exercise is missing. Thus, neuromuscular activation of the diaphragm which in turn promotes optimal activation of the transversus abdominis may further help to address suboptimal respiration and posture which is associated with low back pain. This research work experiment an exercise called, all fours belly breathing exercise with an assumption as it will help in the activation of the diaphragm which in turn promotes optimal activation of the transversus abdominis may further help to address suboptimal respiration and posture.

\section{Materials and Methods}

For this study, 20 participants (8male, 12female and mean age of 20.10) were participated in this study; participants were screened to meet the inclusion criteria of normal subjects. Initially on the first day of first week all the participants were assessed with shuttle run and later participants were training with all fours belly breathing and at the end of fourth week all the participants were reassessed with shuttle run.

\section{Shuttle Run Test}

The 20m Shuttle Run Test (SRT) is used to measure the maximal running aerobic fitness, which are also known as beep or bleep test. It has a total number of twenty-one levels and different number of shuttles for each level according to the speed. Throughout the study, this test was requiring conducting twice. The first SRT was conducted as a pretest after the participants had been chosen. After four weeks of all fours belly breathing exercise, a final Shuttle Run Test (posttest) was held.

\section{Procedure}

a. Before the test started, a 10m distance running track was pre-measured and checked to make sure the flooring and environment was suitable to run.

b. Marking cones were arranged as landmark of the starting and ending points, so that participants were clear about where and when to start and turn during running. c. Every subject was asked to perform a brief warm up for about 5 minutes to prevent injury.

d. Subject was asked to run to and fro between two lines within timing by listening to recorded beeps.

e. At first, the subject stood behind the beginning line and began to run to the second line and returned when instructed by the recorded audio.

f. The initial speed was quite slow, and subject had to continue running and turning between the two lines when signaled by the recording. Among the several versions of the shuttle run test, the commonly used version was chosen in this study. It had an initial running velocity of $8.5 \mathrm{~km} / \mathrm{hr}$, which increased by $0.5 \mathrm{~km} / \mathrm{hr}$ each minute.

g. About one minute later, there was a sound which indicated increase in speed, and this continued every minute (level).

h. When the subject reached the line before the beep sounds, he/she will wait and continue until the beep sounds are heard, where as if the beep sounds came before the subject reached the line, a warning was given and must continue to run to the line, and tried to catch up with the pace within two more 'beeps'.

i. The test was terminated when the subject failed to reach the line (within two meters) for two consecutive ends after a warning or subject intended to stop

j. After termination of test, subjects were required to have a cool down session for around five minutes. Slow walking and some stretching exercises were taught by researcher to prevent delayed onset muscle soreness (DOMS).

\section{Scoring}

The level and number of shuttles $(20 \mathrm{~m})$ reached was recorded before the subject was unable to keep up with the recording. The last level completed was marked down in a prepared beep recording sheet. After 4-week of breathing exercise training, the aerobic fitness of subjects were also measured by Shuttle Run Test. In order to increase validity of the results of study, a Beep Test V02max Calculator developed by to pend Sports from the tables in a published article to calculate predicted V02max. By entering the age, gender, the level and number of shuttles completed, the calculator will generate the results (predicted V02max).

\section{Training Protocol}

All the subjects had participated in 4-weeks of all fours belly breathing training. The training started immediately the day after pre SRT session. During the first week of training, researcher taught and monitored the breathing technique one to one. The following three weeks of training, the twenty subjects 
were divided into four groups for group therapy. To obtain a training response muscles must be overloaded, this overloading can be applied by altering duration, intensity or frequency. For respiratory muscles of a healthy individual, the training should be takes place daily or at least three times per week, and once or twice per day. The suggested intensity is $50-70 \%$ (typically yields failure within 30 breaths), or 2-3 minutes and durations are 30 breaths or 2 to 3 minutes per session [6]. Therefore, in this study, the parameters were standardized to minimize error:

Standard Parameters:

a. intensity $=50-70 \%$

b. duration $=30$ breaths

c. frequency = 3 times/week; twice/day

A mat was set up in front of mirror for all fours belly breathing. By doing in front of mirror, the subjects can get a feedback on their way of performing the breathing technique and able to correct it back immediately. In order to minimize error, along with the explanation of procedures to the subjects, researcher also demonstrated the correct way of the breathing technique.

Instructions for All Fours Belly Breathing:

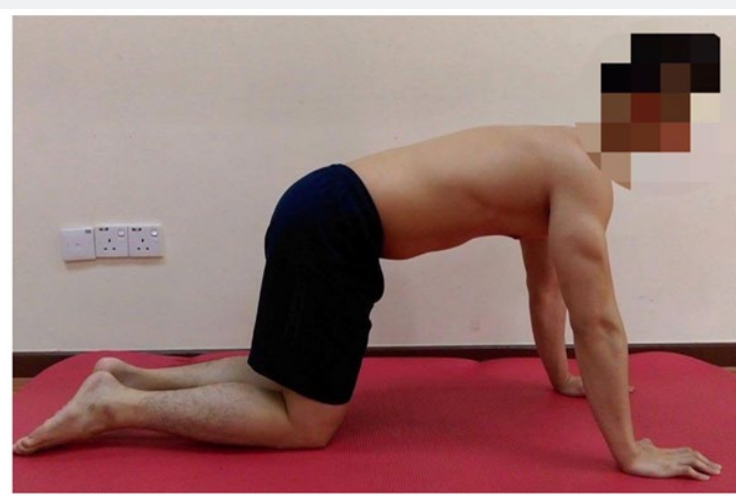

Figure 1: Starting position for all fours belly breathing exercise.

i. $\quad$ Start in quadruped position with slight posterior tilt of pelvis, and place both hands shoulder width apart while both knees pelvis width apart. It should be in 90 degree or perpendicular to the body. Subject's spine should be in neutral position (Figure 1).

ii. Take a deep breath through nose and try to imagine the air is pushing into the stomach, fell the stomach blow up with the air.

iii. While breathing out through mouth, drive the sternum up towards the ceiling to create a hump in the upper back (Figure 2).

iv. Focus the breath and imagine the air get into the dome created and slowly and fully breathed out through mouth.

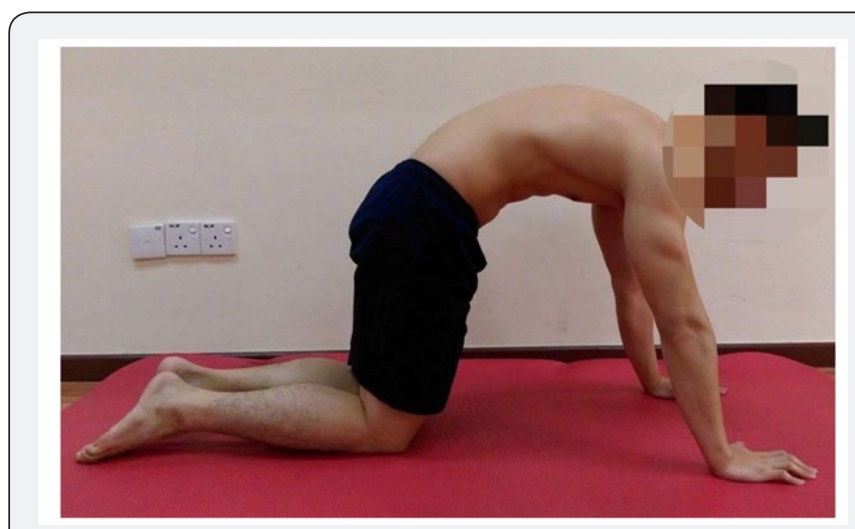

Figure 2: Spinal curvature created for breathing.

v. Maintain the position for 6 breaths and return to the starting position.

vi. Subject was asked to have 1 min resting interval and then repeat the above procedures for 5 cycles.

\section{Precautions}

I. Subjects are not allowed to hold breath throughout the process.

II. One min resting interval is given to prevent hyperventilation.

Statistical Analysis: Student t test was used for analysis of data collected from pre exercise and post exercise training shuttle run rest.

Results: SRT increased from 3.31 to 3.95 of predicted oxygen uptake from 23.78 to 25.87. Although the SRT score doesn't shows much difference whereas the predicted Vo2 max shows reasonable difference (Tables $1 \& 2$ ).

\section{Discussion}

The objective of this study was to identify the effect of 4 weeks of all fours belly breathing exercise on shuttle run performance among normal subjects. All fours belly breathing exercise is defined as a respiratory technique and corrective exercise that performed in a quadruped position, aimed to restore thoracic flexion of and to promote better control of pelvis and ribcage. This new respiratory technique implemented is to fulfill the dearth of breathing technique with activation of all abdominal muscles (RA, IO, EO and TA), as well as diaphragm at the same time. According to length-tension relationship, muscle activated maximally at its optimum length and relatively less force generated at either shorter or longer lengths [7]. "Optimal length-tension relationships, recruitment patterns, and joint motions in core muscles establish neuromuscular efficiency throughout entire human movement system which allows efficient acceleration, deceleration, and stabilization during dynamic movements, as well as prevention of possible injuries [8]." An all fours is a position which the muscles are in relaxed 
state, it is ideally for the respective muscles to get recruited and trained. It is an ideal position for the anterior part of body

(abdominals) to work while the posterior part (excessive tone of extensor muscles) is inhibited.

Table 1: Mean differences between SRT score before (pre) and SRT score after intervention (post).

\begin{tabular}{|c|c|c|c|c|c|}
\hline & Paired samples mean (sd) & Mean difference (sd) & 95\% Confidence Interval & $t$ & P value \\
\hline SRT score before & $3.31(-1.47)$ & -0.65 & & & \\
\hline$(0.56)$ & $-0.91--0.39$ & $-5.23^{*}$ & $<0.001$ & & \\
\hline SRT score after & $3.95(-1.37)$ & & & \\
\hline
\end{tabular}

Table 2: Mean differences between predicted $\mathrm{VO}_{2}$ max before (pre) and predicted $\mathrm{VO}_{2}$ max after intervention (post).

\begin{tabular}{|c|c|c|c|c|c|}
\hline & Paired samples mean (sd) & Mean difference (sd) & 95\% Confidence Interval & t & P value \\
\hline Predicted $\mathrm{VO}_{2}$ max before & 23.78 & -2.09 & & \\
\hline$(1.51)$ & $-2.79 \_-1.38$ & $-6.185^{*}$ & $<0.001$ & \\
\hline Predicted $\mathrm{VO}_{2}$ max after & 25.87 & & & \\
\hline$(4.74)$ & & & & \\
\hline
\end{tabular}

It is believed that all fours belly breathing exercise has many benefits. Firstly, all fours require subjects to hold their spine in neutral position which helps in correcting the excessive (hyper lordosis) or loss of normal lumbar curvature (flat back) of the spine. While in the quadruped position itself, posterior pelvic tilt is achieved. As a breathing technique, all fours belly breathing primarily works on the activation of respiratory muscles like abdominal muscles and diaphragm. While inhaling and making sure that the air goes to the stomach, diaphragm is activated. Then follow by exhaling through mouth and slowly driving the sternum up towards the ceiling to create a dome shape in the upper back. Make sure the belly is drawn in towards the spine to activate the local abdominal muscle. In order to against the gravitational pull, abdominal muscles are activated and strengthened at the same time during this process. Besides, subjects are required to exhale fully which also facilitate the abdominals. Subjects need to maintain this position and breathe in and out for another 5 to 6 breaths. In this position, the posterior mediastinum is expanded and creating a better zone of apposition (ZOA) for diaphragm function. It also helps in restoring the thoracic flexion for people with flat T-spine. On the other hand, pump handle movement of ribcage is relatively increased throughout the proper breathing patterns. By expanding the ribcage posteriorly, there is an increase in the antero-posterior diameter of thoracic cavity. This movement is essential in the mechanism of breathing for the expansion and reduction of the intra-thoracic volume.

The results of our study showed improvement on the SRT score after 4 weeks of training. SRT is one of the field tests developed and widely used to monitor changes in cardiorespiratory fitness. Cardiorespiratory fitness (CRF) essential for both health and fitness and is usually expressed in metabolic equivalents (METs) or maximal oxygen uptake (VO2max). CRF has a close relationship with physical activity. Studies shown regular physical activity can improve CRF of individuals [9-11]. Similarly, the level of CRF depends on several modifiable and non-modifiable causal factors [11] (Table 3).
Among the modifiable factors, physical activity is the primary determinant to the level of CRF. In order to sustain endurance and eliminate waste products during prolonged exercise, both respiratory and circulatory systems play important roles by Lee et al. (2010), Journal of Psychopharmacology, 24(11), p. 32. Copyright 2010 by Lee, et al.)

Table 3: Determinants of cardiorespiratory fitness.

\begin{tabular}{|c|c|}
\hline Non-modifiable & Modifiable \\
\hline Age & Physical activity \\
\hline Gender & Smoking \\
\hline Genotype & Obesity \\
\hline & Medical condition \\
\hline
\end{tabular}

The mean difference between pre and post test score showed the result is statistically significant $(\mathrm{p}<0.001)$. There is an association between all fours belly breathing exercise and shuttle run performance. We can conclude that after 4 weeks of all fours belly breathing exercise, participants had improved in their $\mathrm{CRF}$, as shown in the improvement in $\mathrm{VO}_{2}$ max predicted from the SRT before and after training $(\mathrm{p}<0.001)$. By the results scored in SRT, $\mathrm{VO}_{2}$ max of the individual can be estimated. SRT is one of the field tests developed and widely used to monitor changes in CRF. The results may be affected by several factors:

a. familiarity to the test and instructions given

b. running and turning technique

c. environmental factor like weather and running surface

d. attire and condition

e. motivation and purpose of testing.

In our study, first, instructions were clearly explained to each of the participants and a trial was given for them before running the test. Running and turning techniques were not equally familiarized among all the individuals. Besides, the SRT was conducted indoor and a non-slip running surface. However, 
some of the participants felt that the surrounding air was a limiting factor to the run. They felt breathless easily if keep inhaling the dry air (air-con). All subjects were presented with their sport attires and their health conditions of the day were considered. The purpose of study had been clearly explained to all participants and they were willing to take part. No one was forced or done the test unpleasantly. Some of them were participated with their friends, thus there was a motivation and courage from the peers, which may further increase the performance.

In addition to that, the research question was 'Does all fours belly breathing exercise have the effects on shuttle run performance?' After analyzing the result, we found that male participants have scored higher and had a higher mean difference in the SRT score before and after intervention than females, which shows that all fours belly breathing has more effect on CRF among male participants. As it may due to the gender difference in V02max, with the body mass difference; the anthropometric variables can affect their CRF level. It was proved that the "gender differences in aerobic power were not fully explained by differences in body size $[12,13]$." The CRF of females is lower than males.

\section{Limitations}

There were several limitations in our study. We only recruited small sample size in the study due to the difficulty in recruiting the participants in the short period of time. It is quite difficult to identify the significant relationships between the variables with small sample size. A larger sample size should be recruited in future studies to get a more representative result. In this study, we did not measure the electromyography (EMG) activities of the target muscles due to budget and resources constraint, causing no evidence of the muscle activity. For example, during the study period, we did not know whether the participants had participated in any other physical activity or training that may be one of the confounding variables to our findings. By doing other physical activity, it is believed that there will be some improvement in $\mathrm{CRF}$, which may also secondarily cause subjects to perform better in the post SRT. We did not include a control group that did not receive any respiratory training in this study because this is a pilot study to determine the association between the two variables. After our study proved the effects of all fours belly breathing, other researcher can work in comparing between the effects of two different respiratory trainings.

\section{Conclusion}

This present study concludes that all fours belly breathing improves shuttle run performance among the normal subjects.

\section{References}

1. Aliverti A, Cala SJ, Duranti R (1997) Human respiratory muscle actions and control during exercise. J Appl Physiol (1985) 83(4): 1256-1269.

2. Henke KG, Sharratt M, Pegelow D (1988) Regulation of end-expiratory lung volume during exercise. J Appl Physiol 64(1): 135-146.

3. Stubbing DG, Pengelly LD, Morse JL (1980) Pulmonary mechanics during exercise in normal males. J Appl Physiol 49(3): 506-510.

4. Hodges PW, Eriksson AE, Shirley D (2005) Intra-abdominal pressure increases stiffness of the lumbar spine. J Biomech 38(9): 1873-1880.

5. Hodges PW, Gurfinkel VS, Brumagne S (2002) Coexistence of stability and mobility in postural control: evidence from postural compensation for respiration. Exp Brain Res 144(3): 293-302.

6. McConnell A (2013) Respiratory muscle training: Theory and practice. Edinburgh: Churchill Livingstone

7. Schwartzstein RM, Parker MJ (2006) Respiratory physiology: A clinical Approach, Philadelphia: Lippincott Williams \& Wilkins.

8. Jones AM Carter H (2000) The Effect of Endurance Training on Parameters of Aerobic Fitness. Sports Medicine 29(6): 373-

9. 386 .

10. Armstrong N, Tomkinson G, Ekelund U (2011) Aerobic fitness and its relationship to sport, exercise training and habitual physical activity during youth. Br J Sports Med 45(11): 849-858.

11. Jones J (2016) NASM Core Training Concepts - The Healthy Gamer.

12. Lee D, Artero EG, Sui X, Blair SN (2010) Review: Mortality trends in the general population: The importance of cardiorespiratory fitness. J Psychopharmacol 24(4) 27-35.

13. Rippe JM (2012) Encyclopedia of lifestyle medicine \& health. Thousand Oaks, CA Sage Publications.

Your next submission with Juniper Publishers
will reach you the below assets
- Quality Editorial service
- Swift Peer Review
- Reprints availability
- E-prints Service
- Manuscript Podcast for convenient understanding
- Global attainment for your research
- Manuscript accessibility in different formats
( Pdf, E-pub, Full Text, Audio)
- Unceasing customer service
Track the below URL for one-step submission
https://juniperpublishers.com/online-submission.php

